\title{
Filtering for Networked Stochastic Time-Delay Systems With Sector Nonlinearity
}

\author{
Guoliang Wei, Zidong Wang, Xiao He, and Huisheng Shu
}

\begin{abstract}
This paper is concerned with the filtering problem for a class of discrete-time stochastic nonlinear networked control systems with network-induced incomplete measurements. The incomplete measurements include both the multiple random communication delays and random packet losses, which are modeled by a unified stochastic expression in terms of a set of indicator functions that is dependent on certain stochastic variable. The nonlinear functions are assumed to satisfy the sector nonlinearities. The purpose of the addressed filtering problem is to design a linear filter such that the filtering-error dynamics is exponentially mean-square stable. By using the linear-matrix-inequality (LMI) method and delay-dependent technique, sufficient conditions are derived which are dependent on the occurrence probability of both the random communication delays and missing measurement. The filter gain is then characterized by the solution to a set of LMIs. A simulation example is exploited to demonstrate the effectiveness of the proposed design procedures.
\end{abstract}

Index Terms-Exponential mean-square stability, networked control system (NCS), random communication delay, random packet loss, sector nonlinearity, stochastic disturbance.

\section{INTRODUCTION}

W ITH THE increasing application of networks in the complex dynamical processes such as advanced aircraft, spacecraft, automotive, and manufacturing processes, the networked control systems (NCSs) are currently receiving considerable attention in the literature as illustrated by recent articles (see, e.g., [8], [12] and references therein). Communication delays (or networked-induced delays) and missing measurements (or packet losses) have become unavoidable that constitute the main causes for degrading the achievable performance of the networked systems. Therefore, in the past decade, the filtering and control problems with communication delays and/or missing measurements have been extensively considered by many researchers (see, e.g., [14], [15]).

Unfortunately, in most of existing references, the communication delay and the data-missing problems have been studied

Manuscript received May 09, 2008; revised August 26, 2008. Current version published January 16, 2009. This work was supported in part by the Engineering and Physical Sciences Research Council (EPSRC) of the U.K. under Grant GR/S27658/01, an International Joint Project sponsored by the Royal Society of the United Kingdom, by the Shanghai Natural Science Foundation of China under Grant 07ZR14002, by the National Natural Science Foundation of China under Grant 60874113, and by the Alexander von Humboldt Foundation of Germany. This paper was recommended by Associate Editor W. X. Zheng.

G. Wei and H. Shu are with the School of Information Science and Technology, Donghua University, Shanghai 200051, China.

Z. Wang is with the Department of Information Systems and Computing, Brunel University, Uxbridge UB8 3PH, U.K., and also with the School of Information Science and Technology, Donghua University, Shanghai 200051, China (e-mail: Zidong.Wang@brunel.ac.uk).

$\mathrm{X}$. He is with the Department of Automation, Tsinghua University, Beijing 100084, China.

Digital Object Identifier 10.1109/TCSII.2008.2008522 separately by using different models, despite the fact that both problems are caused by the limited bandwidth of communication networks and may be present simultaneously. Compared to large amount of results for linear deterministic networked systems, the nonlinear Itô-type stochastic NCSs have received much less attention mainly due to the mathematical complexity [11].

On the one hand, Itô-type stochastic systems (also referred to as stochastic systems with multiplicative noise) have come to play an important role in practical applications such as chemistry, biology, ecology, control, and information systems [7]. On the other hand, the sectorlike nonlinearities usually occur in practical systems that experience harsh environments such as uncontrollable elements (e.g., variations in flow rates, temperature, etc.) and aggressive conditions (e.g., corrosion, erosion, fouling, etc.), which give rise to possible performance degradation (see, e.g., [1], [2]). It is well known that the filtering problem is one of the fundamental problems in control and signal processing that has attracted renewing research attention following the celebrated Kalman filtering scheme [3]-[7], [10], [12], [14], [15]. However, to the best of the authors' knowledge, the filtering problem for Itô-type stochastic networked time-delay systems with simultaneous random occurrences of communication delays and missing measurements has not been fully investigated yet, which still remains as a challenging research issue.

In this paper, the filtering problem is addressed for a class of discrete-time stochastic nonlinear networked systems with multiple random communication delays and random packet losses. The communication-delay and packet-loss problems, which are frequently encountered in communication networks with limited digital capacity, are modeled by a stochastic mechanism that combines a certain set of indicator functions dependent on the same stochastic variable. We first derive sufficient conditions dependent on the occurrence probabilities of both the random communication delay and missing measurement, which ensure the desired filtering performance for the networked system. Then, we characterize the filter gain in terms of the solution to a set of linear matrix inequalities (LMIs). A simulation example is exploited to demonstrate the effectiveness of the proposed design procedures.

\section{PROBlem Formulation}

Consider the following discrete-time stochastic nonlinear NCS with multiple delays in the state:

$$
\begin{aligned}
(\Sigma): x(k+1) & =A x(k)+\sum_{i=1}^{q} B_{i} \phi_{i}\left(x\left(k-d_{i}\right)\right)+\operatorname{Ex}(k) \omega(k) \\
x(k) & =\mu(k), \quad k=-d_{q},-d_{q}+1, \ldots, 0
\end{aligned}
$$


where $x(k) \in \mathbb{R}^{n}$ is the state and $d_{i} \in \mathbb{Z}^{+}(i=1, \ldots, q)$ are known constant time delays that are assumed to satisfy $d_{1}<$ $d_{2}<\cdots<d_{q}$ for simplicity. $\omega(k)$ is a 1-D Gaussian white noise sequence satisfying $\mathbb{E}\{\omega(k)\}=0$ and $\mathbb{E}\left\{\omega^{2}(k)\right\}=\delta^{2}$. $\phi_{i}(\cdot)$ is the sector nonlinearity, and $\mu(k)$ is the initial state of the system.

The measurement with random communication delays and random data missing is described as

$$
\begin{aligned}
y(k)=I_{\left\{\tau_{k}=0\right\}} C x(k)+\sum_{i=1}^{q} I_{\left\{\tau_{k}=d_{i}\right\}} D_{i} \theta_{i}( & \left.x\left(k-d_{i}\right)\right) \\
& +F x(k) \omega(k)
\end{aligned}
$$

where $y(k) \in \mathbb{R}^{p}$ is the measured output vector. For the indicator functions $I_{\left\{\tau_{k}=0\right\}}$ and $I_{\left\{\tau_{k}=d_{i}\right\}}$, we assume that $\mathbb{E}\left\{I_{\left\{\tau_{k}=0\right\}}\right\}=\operatorname{Pr}\left\{\tau_{k}=0\right\}=p_{0}$ and $\mathbb{E}\left\{I_{\left\{\tau_{k}=i\right\}}\right\}=\operatorname{Pr}\left\{\tau_{k}=d_{i}\right\}=p_{i}$, where $p_{i}(i=0,1,2, \ldots, q)$ are known positive scalars and $\sum_{j=0}^{q} p_{j} \leq 1 . \tau_{k}$ is a mutually independent stochastic variable sequence used to describe, at time $k$, the occurred delays and data missing of the measured output information. $\theta_{i}(\cdot)$ represents the sector nonlinearity. $A$, $C, E, F, B_{i}$, and $D_{i}(i=1, \ldots, q)$ are constant matrices with appropriate dimensions.

Remark 1: The indicator functions in (3) dependent on a stochastic variable sequence $\tau_{k}$ are utilized to describe the two kinds of incomplete measurements (random communication delays and random data missing) simultaneously. That is, if $\sum_{j=0}^{q} p_{j}=1$, then no data missing occurs in the system; and if $\sum_{j=0}^{q=0} p_{j}<1$, the data-missing occurrence probability is $1-\sum_{j=0}^{q} p_{j}$, where $p_{0}$ and $p_{i}(i=1, \ldots, q)$ denote the occurrence probability of the data missing and the communication delay $d_{i}$ in the networked system, respectively. These two kinds of incomplete measurements are induced by the limited bandwidth of communication networks, and therefore, they should be considered at the same time as done in (3). This model can be regarded as an extension of those adopted in [14] and [15] and has been used to deal with a robust filtering problem for a class of linear deterministic discrete-time NCSs in [3].

Assumption 1: The nonlinear functions $\phi_{i s}(\cdot)$ (the sth element of $\phi_{i}(\cdot)$ ) and $\theta_{i s}(\cdot)$ (the sth element of $\phi_{i}(\cdot)$ ) in stochastic system (1)-(3) satisfy the following sector conditions for $\forall x_{s} \in \mathbb{R}, x_{s} \neq 0, i=1, \ldots, q, s=1, \ldots, n$ :

$$
\begin{aligned}
& 0 \leq \varphi_{i s}\left(x_{s}\right):=\frac{\phi_{i s}\left(x_{s}\right)}{x_{s}} \leq k_{i s} \\
& 0 \leq \vartheta_{i s}\left(x_{s}\right):=\frac{\theta_{i s}\left(x_{s}\right)}{x_{s}} \leq l_{i s}
\end{aligned}
$$

which are equivalent to the following matrix inequalities:

$$
\begin{aligned}
\phi_{i}(x)\left(\phi_{i}(x)-K_{i} x\right) & \leq 0 \\
\theta_{i}(x)\left(\theta_{i}(x)-L_{i} x\right) & \leq 0
\end{aligned}
$$

where $\phi_{i}(x)=\left[\begin{array}{lll}\phi_{i 1}\left(x_{1}\right) & \cdots & \phi_{\text {in }}\left(x_{n}\right)\end{array}\right]^{\mathrm{T}}, \theta_{i}(x)=$ $\left[\theta_{i 1}\left(x_{1}\right) \quad \cdots \quad \theta_{\text {in }}\left(x_{n}\right)\right]^{\mathrm{T}}, K_{i}=\operatorname{diag}\left\{k_{i 1}, k_{i 2}, \ldots, k_{\text {in }}\right\}>0$, and $L_{i}=\operatorname{diag}\left\{l_{i 1}, l_{i 2}, \ldots, l_{\text {in }}\right\}>0$.

Remark 2: The sector nonlinearities resulting from the complex environments exist widely in practical systems and often lead to the poor performance of the controlled system. Many researchers have investigated the analysis and synthesis prob- lems for various systems with sectorlike nonlinearities (see [1], [2]).

In this paper, we are interested in designing a filter of the following structure:

$$
\left(\Sigma_{f}\right): \hat{x}(k+1)=G \hat{x}(k)+H y(k)
$$

where $\hat{x}(t) \in \mathbb{R}^{n}$ is the state estimate and $G$ and $H$ are filter parameters to be determined.

By augmenting the state variables

$$
\xi(k):=\left[\begin{array}{l}
x(k) \\
\hat{x}(k)
\end{array}\right] \quad \sigma_{d i}(k):=P\left[\begin{array}{c}
\phi_{i}\left(x\left(k-d_{i}\right)\right) \\
\theta_{i}\left(x\left(k-d_{i}\right)\right)
\end{array}\right]
$$

and combining $(\Sigma)$ and $\left(\Sigma_{f}\right)$, we obtain the filtering-error dynamics as follows:

$$
\begin{aligned}
\xi(k+1)=\bar{A} \xi(k)+ & \sum_{i=1}^{q} \bar{B}_{i} \sigma_{d i}(k)+I_{\tau_{k}}^{0} \bar{C} Z \xi(k) \\
& +\sum_{i=1}^{q} I_{\tau_{k}}^{i} \bar{D}_{i} \sigma_{d i}(k)+\bar{E} Z \xi(k) \omega(k)
\end{aligned}
$$

where $I_{\tau_{k}}^{i}=I_{\left\{\tau_{k}=i\right\}}-p_{i}(i=0,1, \ldots, q)$ and

$$
\begin{array}{ll}
\bar{A}=\left[\begin{array}{cc}
A & 0 \\
p_{0} H C & G
\end{array}\right] & \bar{B}_{i}=\left[\begin{array}{cc}
B_{i} & 0 \\
0 & p_{i} H D_{i}
\end{array}\right] \\
\bar{C}=\left[\begin{array}{c}
0 \\
H C
\end{array}\right] & \bar{D}_{i}=\left[\begin{array}{cc}
0 & 0 \\
0 & H D_{i}
\end{array}\right] \\
\bar{E}=\left[\begin{array}{c}
E \\
H F
\end{array}\right] & Z=\left[\begin{array}{ll}
I & 0
\end{array}\right] .
\end{array}
$$

It follows from (1), (3), (4), and (7) that

$$
\begin{aligned}
\xi(k+1)=\bar{A} \xi(k)+ & \sum_{i=1}^{q} \bar{B}_{i} \varsigma_{d i}(k) Z \xi_{d i}(k)+I_{\tau_{k}}^{0} \bar{C} Z \xi(k) \\
& +\sum_{i=1}^{q} I_{\tau_{k}}^{i} \bar{D}_{i} \sigma_{d i}(k)+\bar{E} Z \xi(k) \omega(k)
\end{aligned}
$$

where

$$
\begin{aligned}
\xi_{d i}(k) & :=\left[\begin{array}{l}
x\left(k-d_{i}\right) \\
\hat{x}\left(k-d_{i}\right)
\end{array}\right] \\
\varsigma_{d i}(k) & :=\left[\begin{array}{l}
\left.\operatorname{diag}\left\{\bar{\varphi}_{d i 1}(k), \ldots, \bar{\varphi}_{\operatorname{din}}(k)\right)\right\} \\
\left.\operatorname{diag}\left\{\bar{\vartheta}_{d i 1}(k), \ldots, \bar{\vartheta}_{\operatorname{din}}(k)\right)\right\}
\end{array}\right]
\end{aligned}
$$

with $\bar{\varphi}_{d i j}(k):=\varphi_{i j}\left(x\left(k-d_{i}\right)\right.$ and $\bar{\vartheta}_{d i j}(k):=\vartheta_{i j}(x(k-$ $\left.d_{i}\right)(j=1, \ldots, n)$.

Observe the system (7) and let $\xi(k ; \nu)$ denote the state trajectory from the initial data $\xi(\theta)=\nu(s)$ on $-d_{k} \leq s \leq 0$. Obviously, $\xi(k, 0) \equiv 0$ is the trivial solution of system (7), corresponding to the initial data $\nu=0$.

Definition 1: For the system (7) and every initial conditions $\nu$, the trivial solution is said to be exponentially mean-square stable if there exist scalars $\alpha(\alpha>0)$ and $\beta(0<\beta<1)$ such that

$$
\mathbb{E}|\xi(k, \nu)|^{2} \leq \alpha \beta^{k} \sup _{-d_{q} \leq s \leq 0} \mathbb{E}|\nu(s)|^{2} .
$$

The purpose of this paper is to design a filter of the form (6) for the system (1)-(3) such that, for all admissible random communication time delays, random data missing, and exogenous 
stochastic disturbances, the filtering-error system (7) is exponentially mean-square stable.

\section{MAIN RESULTS}

Theorem 1: Consider the filtering-error system (7) with given filter parameters. If there exist positive-definite matrices $P>0$, $Q_{j}>0, S_{j}>0, M_{j}>0$, and $T_{j}>0(j=1, \ldots, q)$ such that the following matrix inequalities:

$$
\begin{aligned}
& {\left[\begin{array}{cccccc}
\Omega_{1} & -M_{d} & 0 & \Omega_{2} & 0 & \Omega_{5} \\
* & -Q_{d} & \bar{K}_{d}^{\mathrm{T}} & 0 & 0 & 0 \\
* & * & -I_{d} & \Omega_{3} & \Omega_{4} & 0 \\
* & * & * & -\Lambda_{1} & 0 & 0 \\
* & * & * & * & -P_{d} & 0 \\
* & * & * & * & * & -\Lambda_{2}
\end{array}\right]<0} \\
& {\left[\begin{array}{cc}
S_{j} & M_{j} \\
* & T_{j}
\end{array}\right]>0 \quad \forall j=1, \ldots, q}
\end{aligned}
$$

hold, where

$$
\begin{aligned}
\Omega_{1} & :=-P+\sum_{j=1}^{q} Z^{\mathrm{T}} Q_{j} Z+\sum_{j=1}^{q}\left(Z^{\mathrm{T}} M_{j}^{\mathrm{T}}+M_{j} Z\right)+\sum_{j=1}^{q} d_{j} S_{j} \\
\Omega_{2} & :=\left[\left(\bar{A}^{\mathrm{T}}-I\right) Z^{\mathrm{T}} \Pi \bar{A}^{\mathrm{T}} P\right] \\
\Omega_{3} & :=\left[\bar{B}_{d}^{\mathrm{T}} Z^{\mathrm{T}} \Pi \bar{B}_{d}^{\mathrm{T}} P\right] \\
\Omega_{4} & :=\rho_{d} \bar{D}_{d}^{\mathrm{T}} P \\
\Omega_{5} & :=\left[\delta Z^{\mathrm{T}} \bar{E}^{\mathrm{T}} Z^{\mathrm{T}} \Pi \rho_{0} Z^{\mathrm{T}} \bar{C}^{\mathrm{T}} P \delta Z^{\mathrm{T}} \bar{E}^{\mathrm{T}} P \bar{A}^{\mathrm{T}} P\right] \\
P_{d} & :=\operatorname{diag}_{q}\{P\} \\
\Pi & :=\sum^{q} d_{j} T_{j} \\
I_{d} & :=\operatorname{diag}\{I\} \\
M_{d} & :=\left[M_{1}, \ldots, M_{q}\right] \\
Q_{d} & :=\operatorname{diag}\left\{Q_{1}, \ldots, Q_{q}\right\} \\
\bar{K}_{d} & :=\operatorname{diag}\left\{\bar{K}_{1}^{\mathrm{T}}, \ldots, \bar{K}_{q}^{\mathrm{T}}\right\} \\
\bar{K}_{j}^{\mathrm{T}} & :=\left[K_{j} L_{j}\right] \\
\bar{B}_{d} & :=\left[\bar{B}_{1}, \ldots, \bar{B}_{q}\right] \\
\bar{D}_{d} & :=\operatorname{diag}\left\{\bar{D}_{1}, \ldots, \bar{D}_{q}\right\} \\
\rho_{j} & :=\sqrt{p_{j}} ; \rho_{d}:=\operatorname{diag}\left\{\rho_{1}, \ldots, \rho_{q}\right\} ; \rho_{0}:=\sqrt{p_{0}} \\
\Lambda_{1} & :=\operatorname{diag}\{\Pi, P\} ; \Lambda_{2}:=\operatorname{diag}\{\Pi, P, P, P\}
\end{aligned}
$$

then the system (7) is exponentially mean-square stable.

Proof: Recalling (7), we can write

$$
\begin{aligned}
\xi_{d i}(k) & =\xi(k)-\sum_{m=k-d_{i}}^{k-1}(\xi(m+1)-\xi(m)) \\
& =\xi(k)-\sum_{m=k-d_{i}}^{k-1} \zeta(m)
\end{aligned}
$$

where

$$
\begin{array}{r}
\zeta(m):=(\bar{A}-I) \xi(m)+\sum_{i=1}^{q} \bar{B}_{i} \sigma_{d i}(m)+I_{\tau_{m}}^{0} \bar{C} Z \xi(m) \\
+\sum_{i=1}^{q} I_{\tau_{m}}^{i} \bar{D}_{i} \sigma_{d i}(m)+\bar{E} Z \xi(m) \omega(m) .
\end{array}
$$

By substituting (14) into (8), we can obtain

$$
\begin{aligned}
\xi(k+1)= & \left(\bar{A}+\sum_{i=1}^{q} \bar{B}_{i} \varsigma_{d i}(k) Z\right) \xi(k) \\
& -\sum_{j=1}^{q} \sum_{m=k-d_{j}}^{k-1} \bar{B}_{j} \varsigma_{d j}(k) Z \zeta(m)+I_{\tau_{k}}^{0} \bar{C} Z \xi(k) \\
& +\sum_{i=1}^{q} I_{\tau_{k}}^{i} \bar{D}_{i} \sigma_{d i}(k)+\bar{E} Z \xi(k) \omega(k)
\end{aligned}
$$

Define the following Lyapunov functional candidate for the system (16):

$$
\begin{aligned}
V(\tilde{\xi}(k), k)= & V_{1}(\tilde{\xi}(k), k)+V_{2}(\tilde{\xi}(k), k)+V_{3}(\tilde{\xi}(k), k) \\
= & \xi^{\mathrm{T}}(k) P \xi(k)+\sum_{j=1}^{q} \sum_{m=k-d_{j}}^{k-1} \xi^{\mathrm{T}}(m) Z^{\mathrm{T}} Q_{j} Z \xi(m) \\
& +\sum_{j=1}^{q} \sum_{r=-d_{j}}^{-1} \sum_{m=k+r}^{k-1} \zeta^{\mathrm{T}}(m) Z^{\mathrm{T}} T_{j} Z \zeta(m)
\end{aligned}
$$

where $\tilde{\xi}(k):=\left[\begin{array}{llll}\xi^{\mathrm{T}}(k) & \xi^{\mathrm{T}}(k-1) & \cdots & \xi^{\mathrm{T}}(0)\end{array}\right]^{\mathrm{T}}$.

Calculating the difference of the Lyapunov functional candidate for the system (17) according to (7) gives

$\Delta V(\tilde{\xi}(k), k)=\mathbb{E}\{V(\tilde{\xi}(k+1), k+1) \mid \tilde{\xi}(k)\}-V(\tilde{\xi}(k), k)$.

First, we obtain

$$
\begin{aligned}
& \Delta V_{1}(\tilde{\xi}(k), k) \\
& =\xi^{\mathrm{T}}(k)\left(\bar{A}+\sum_{i=1}^{q} \bar{B}_{i} \varsigma_{d i}(k) Z\right)^{\mathrm{T}} P \\
& \times\left(\bar{A}+\sum_{i=1}^{q} \bar{B}_{i} \varsigma_{d i}(k) Z\right) \xi(k)-\xi^{\mathrm{T}}(k) P \xi(k) \\
& +\left[\sum_{j=1}^{q} \sum_{m=k-d_{j}}^{k-1} \bar{B}_{j} \varsigma_{d j}(k) Z \zeta(m)\right]^{\mathrm{T}} P \\
& \times\left[\sum_{j=1}^{q} \sum_{m=k-d_{j}}^{k-1} \bar{B}_{j} \varsigma_{d j}(k) Z \zeta(m)\right] \\
& -2 \sum_{j=1}^{q} \sum_{m=k-d_{j}}^{k-1} \xi^{\mathrm{T}}(k)\left(\bar{A}+\sum_{i=1}^{q} \bar{B}_{i} \varsigma_{d i}(k) Z\right)^{\mathrm{T}} \\
& \times P \bar{B}_{j} \varsigma_{d j}(k) Z \zeta(m) \\
& +\mathbb{E}\left\{\left[I_{\tau_{k}}^{0} \bar{C} Z \xi(k)+\sum_{i=1}^{q} I_{\tau_{k}}^{i} \bar{D}_{i} \sigma_{d i}(k)\right]^{\mathrm{T}} P\right. \\
& \left.\times\left[I_{\tau_{k}}^{0} \bar{C} Z \xi(k)+\sum_{i=1}^{q} I_{\tau_{k}}^{i} \bar{D}_{i} \sigma_{d i}(k)\right]\right\} \\
& +\mathbb{E}\left\{\omega^{\mathrm{T}}(k) \xi^{\mathrm{T}}(k) Z^{\mathrm{T}} \bar{E}^{\mathrm{T}} P \bar{E} Z \xi(k) \omega(k)\right\} .
\end{aligned}
$$


From Moon's inequality [9], we have

$$
\begin{aligned}
- & 2 \xi^{\mathrm{T}}(k)\left(\bar{A}+\sum_{i=1}^{q} \bar{B}_{i} \varsigma_{d i}(k) Z\right)^{\mathrm{T}} P \bar{B}_{j} \varsigma_{d j}(k) Z \zeta(m) \\
& \leq \xi^{\mathrm{T}}(k) S_{j} \xi(k)+2 \zeta^{\mathrm{T}}(m) Z^{\mathrm{T}} M_{j}^{\mathrm{T}} \xi(k) \\
& -2 \zeta^{\mathrm{T}}(m) Z^{\mathrm{T}} \varsigma_{d j}(k)^{\mathrm{T}} \bar{B}_{j}^{\mathrm{T}} P\left(\bar{A}+\sum_{i=1}^{q} \bar{B}_{i} \varsigma_{d i}(k) Z\right) \xi(k) \\
& +\zeta^{\mathrm{T}}(m) Z^{\mathrm{T}} T_{j} Z \zeta(m)
\end{aligned}
$$

with $0 \leq S_{j}^{\mathrm{T}}=S_{j} \in \mathbb{R}^{2 n \times 2 n}, M_{j} \in \mathbb{R}^{2 n \times n}, 0 \leq T_{j}^{\mathrm{T}}=T_{j} \in$ $\mathbb{R}^{n \times n}$, satisfying (12).

It follows from (5) and the notation of $\sigma_{d i}(k)$ that

$$
\sum_{i=1}^{q} \sigma_{d i}^{\mathrm{T}}(k)\left(\sigma_{d i}(k)-\bar{K}_{j} Z \xi_{d j}(k)\right) \leq 0
$$

with $\bar{K}_{j}$ defined in (13).

Again, we can obtain from the expression (3) that, for $0 \leq$ $i \leq q$ and $0 \leq j \leq q$

$$
\mathbb{E}\left\{\left(I_{\tau_{k}}^{i}\right)\left(I_{\tau_{k}}^{j}\right)\right\}= \begin{cases}p_{i}\left(1-p_{i}\right), & i=j \\ -p_{i} p_{j}, & i \neq j .\end{cases}
$$

In addition, from (17), it follows that

$$
\begin{aligned}
\Delta V_{2}(\tilde{\xi}(k), k)= & \sum_{j=1}^{q} \xi^{\mathrm{T}}(k) Z^{\mathrm{T}} Q_{j} Z \xi(k) \\
& -\sum_{j=1}^{q} \xi_{d j}(k)^{\mathrm{T}} Z^{\mathrm{T}} Q_{j} Z \xi_{d j}(k) \\
\Delta V_{3}(\tilde{\xi}(k), k)= & \sum_{j=1}^{q} d_{j} \zeta^{\mathrm{T}}(k) Z^{\mathrm{T}} T_{j} Z \zeta(k) \\
& -\sum_{j=1}^{q} \sum_{r=k-d_{j}}^{k-1} \zeta^{\mathrm{T}}(r) Z^{\mathrm{T}} T_{j} Z \zeta(r) .
\end{aligned}
$$

From (15) and (19)-(24), it can be seen that

$$
\begin{aligned}
\Delta V( & \xi(k), k) \\
\leq & \xi^{\mathrm{T}}(k)\left[\bar{A}^{\mathrm{T}} P \bar{A}-P+p_{0} Z^{\mathrm{T}} \bar{C}^{\mathrm{T}} P \bar{C} Z\right] \xi(k) \\
& +\left(\sum_{j=1}^{q} \bar{B}_{j} \sigma_{d j}(k)\right)^{\mathrm{T}} P\left(\sum_{j=1}^{q} \bar{B}_{j} \sigma_{d j}(k)\right) \\
& +\sum_{i=1}^{q} \sigma_{d i}^{\mathrm{T}}(k)\left(p_{i} \bar{D}_{i}^{\mathrm{T}} P \bar{D}_{i}-2 I\right) \sigma_{d i}(k) \\
& +\delta^{2} \xi^{\mathrm{T}}(k) Z^{\mathrm{T}} \bar{E}^{\mathrm{T}} P \bar{E} Z \xi(k) \\
& +\sum_{j=1}^{q}\left[\xi^{\mathrm{T}}(k) Z^{\mathrm{T}} Q_{j} Z \xi(k)-\xi_{d j}^{\mathrm{T}}(k) Z^{\mathrm{T}} Q_{j} Z \xi_{d j}(k)\right] \\
& +2 \sum_{j=1}^{q} \sigma_{d j}^{\mathrm{T}}(k)\left[\bar{B}_{j}^{\mathrm{T}} P \bar{A} \xi(k)+\bar{K}_{j} Z \xi_{d j}(k)\right]
\end{aligned}
$$

$$
\begin{aligned}
& +\sum_{j=1}^{q} d_{j} \xi^{\mathrm{T}}(k) S_{j} \xi(k)+\sum_{j=1}^{q} d_{j} \zeta^{\mathrm{T}}(k) Z^{\mathrm{T}} T_{j} Z \zeta(k) \\
& +2 \sum_{j=1}^{q} \xi^{\mathrm{T}}(k) Z^{\mathrm{T}} M_{j}^{\mathrm{T}} \xi(k)-2 \sum_{j=1}^{q} \xi_{d j}^{\mathrm{T}}(k) M_{j}^{\mathrm{T}} \xi(k) \\
& -\left[p_{0} \bar{C} Z \xi(k)+\sum_{i=1}^{q} p_{i} \bar{D}_{i} \sigma_{d i}(k)\right]^{\mathrm{T}} P \\
& \times\left[p_{0} \bar{C} Z \xi(k)+\sum_{i=1}^{q} p_{i} \bar{D}_{i} \sigma_{d i}(k)\right] \\
& \leq \bar{\xi}^{\mathrm{T}}(k) \Gamma \bar{\xi}(k)
\end{aligned}
$$

where

$$
\begin{aligned}
& \bar{\xi}(k)=\left[\begin{array}{llllll}
\xi^{\mathrm{T}}(k) & \xi_{d 1}^{\mathrm{T}}(k) Z^{\mathrm{T}} & \cdots & \xi_{d q}^{\mathrm{T}}(k) Z^{\mathrm{T}} \sigma_{d 1}^{\mathrm{T}}(k) & \cdots & \sigma_{d q}^{\mathrm{T}}(k)
\end{array}\right]^{\mathrm{T}} \\
& \Gamma:=\left[\begin{array}{ccc}
\Gamma_{1} & -M_{d} & \Gamma_{2} \\
* & -Q_{d} & \bar{K}_{d}^{\mathrm{T}} \\
* & * & \Gamma_{3}
\end{array}\right]
\end{aligned}
$$

with

$$
\begin{aligned}
\Gamma_{1}:= & \Omega_{1}+\bar{A}^{\mathrm{T}} P \bar{A}+\rho_{0}^{2} Z^{\mathrm{T}} \bar{C}^{\mathrm{T}} P \bar{C} Z+\delta^{2} Z^{\mathrm{T}} \bar{E}^{\mathrm{T}} P \bar{E} Z \\
& +\rho_{0}^{2} Z^{\mathrm{T}} \bar{C}^{\mathrm{T}} Z^{\mathrm{T}} \Pi Z \bar{C} Z+\delta^{2} Z^{\mathrm{T}} \bar{E}^{\mathrm{T}} Z^{\mathrm{T}} \Pi Z \bar{E} Z \\
& +\left(\bar{A}^{\mathrm{T}}-I\right) Z^{\mathrm{T}} \Pi Z(\bar{A}-I) \\
\Gamma_{2}:= & \left(\bar{A}^{\mathrm{T}}-I\right) Z^{\mathrm{T}} \Pi Z \bar{B}_{d}+\bar{A}^{\mathrm{T}} P \bar{B}_{d} \\
\Gamma_{3}:= & \bar{B}_{d}^{\mathrm{T}}\left(Z^{\mathrm{T}} \Pi Z+P\right) \bar{B}_{d}-I_{d} \\
& +\operatorname{diag}\left\{\rho_{1}^{2} \bar{D}_{1}^{\mathrm{T}}\left(P+Z^{\mathrm{T}} \Pi Z\right) \bar{D}_{1}, \ldots, \rho_{q}^{2} \bar{D}_{q}^{\mathrm{T}}\right. \\
& \left.\quad \times\left(P+Z^{\mathrm{T}} \Pi Z\right) \bar{D}_{q}\right\}
\end{aligned}
$$

and $Q_{d}, \bar{K}_{d}, Y_{d}, \bar{B}_{d}, \Pi, I_{d}, \rho_{0}$, and $\rho_{j}(j=1, \ldots, q)$ are defined in (13).

By Schur complement, we can obtain from (11) and (12) that $\Gamma<0$, and therefore, there always exists a scalar $\alpha>0$ such that $\Gamma<\operatorname{diag}\{-\alpha I, 0,0\}$, and subsequently

$\mathbb{E}\{V(\tilde{\xi}(k+1), k+1) \mid \tilde{\xi}(k)\}-V(\tilde{\xi}(k), k)<-\alpha|\xi(k)|^{2}$

Finally, we can confirm from [15, Lemma 1 ] that the filteringerror systems (7) is exponentially stable.

The following theorem shows that the desired filter parameters can be derived by solving several LMIs. The proof is omitted to keep the paper concise.

Theorem 2: Consider the system (7). If there exist matrices $X>0, \mathcal{Y}>0, Q_{j}>0, \bar{S}_{j i}>0, T_{j}>0, \tilde{G}, \tilde{H}, \bar{M}_{j 1}$, and $\bar{M}_{j 2}(j=1,2, \ldots, q ; i=1,2,3)$ such that the following LMIs:

$$
\begin{aligned}
& {\left[\begin{array}{cccccc}
-\Xi_{1} & \Xi_{2} & -\tilde{M}_{d 1} & 0 & \Xi_{4} & \Xi_{7} \\
* & -\Xi_{3} & -\tilde{M}_{d 2} & 0 & \Xi_{5} & \Xi_{8} \\
* & * & -Q_{d} & \bar{K}_{d}^{\mathrm{T}} & 0 & 0 \\
* & * & * & -I_{d} & \Xi_{6} & 0 \\
* & * & * & * & -\Theta_{1} & 0 \\
* & * & * & * & * & -\Theta_{2}
\end{array}\right]<0} \\
& {\left[\begin{array}{ccc}
\bar{S}_{j 1} & \bar{S}_{j 2} & \bar{M}_{j 1} \\
* & \bar{S}_{j 3} & \bar{M}_{j 2} \\
* & * & T_{j}
\end{array}\right]>0 \quad \forall j=1, \ldots, q}
\end{aligned}
$$


hold, where

$$
\begin{aligned}
& \Xi_{1}:=\mathcal{Y}-\Sigma_{j=1}^{q}\left(Q_{j}+d_{j} \bar{S}_{j 1}+\Sigma_{s=1}^{2}\left(\bar{M}_{j s}+\bar{M}_{j s}^{\mathrm{T}}\right)\right) \\
& \Xi_{2}:=\mathcal{Y}+\Sigma_{j=1}^{q}\left(Q_{j}+d_{j} \bar{S}_{j 2}+\bar{M}_{j 1}+\bar{M}_{j 1}^{\mathrm{T}}+\bar{M}_{j 2}\right) \\
& \Xi_{3}:=X-\Sigma_{j=1}^{q}\left(Q_{j}+d_{j} \bar{S}_{j 3}+\bar{M}_{j 1}+\bar{M}_{j 1}^{\mathrm{T}}\right) \\
& \Xi_{4}:=\left[\left(A^{\mathrm{T}}-I\right) \Pi\right)\left[A^{\mathrm{T}} \mathcal{Y} A^{\mathrm{T}} X+p_{0} C^{\mathrm{T}} \tilde{H}^{\mathrm{T}}+\tilde{G}^{\mathrm{T}}\right] \\
& \left.0 \delta E^{\mathrm{T}} \Pi\left[0 \rho_{0} C^{\mathrm{T}} \tilde{H}^{\mathrm{T}}\right]\right] \\
& \Xi_{5}:=\left[\left(A^{\mathrm{T}}-I\right) \Pi\right)\left[A^{\mathrm{T}} \mathcal{Y} A^{\mathrm{T}} X+p_{0} C^{\mathrm{T}} \tilde{H}^{\mathrm{T}}\right] \\
& \left.0 \delta E^{\mathrm{T}} \Pi\left[0 \rho_{0} C^{\mathrm{T}} \tilde{H}^{\mathrm{T}}\right]\right] \\
& \Xi_{6}:=\left[\tilde{B}_{d}^{\mathrm{T}} \Pi \hat{B}_{d} \tilde{D}_{d} 0\left[\begin{array}{ll}
0 & 0
\end{array}\right]\right. \\
& \Xi_{7}:=\left[\left[\delta E^{\mathrm{T}} \mathcal{Y} \delta E^{\mathrm{T}} X+\delta F^{\mathrm{T}} \tilde{H}^{\mathrm{T}}\right]\right. \\
& \left.\left[A^{\mathrm{T}} \mathcal{Y} A^{\mathrm{T}} X+p_{0} C^{\mathrm{T}} \tilde{H}^{\mathrm{T}}+\tilde{G}^{\mathrm{T}}\right]\right] \\
& \Xi_{8}:=\left[\left[\delta E^{\mathrm{T}} \mathcal{Y} \delta E^{\mathrm{T}} X+\delta F^{\mathrm{T}} \tilde{H}^{\mathrm{T}}\right]\left[A^{\mathrm{T}} \mathcal{Y} A^{\mathrm{T}} X+p_{0} C^{\mathrm{T}} \tilde{H}^{\mathrm{T}}\right]\right] \\
& \hat{B}_{d}:=\left[\hat{B}_{1}, \ldots, \hat{B}_{q}\right]^{\mathrm{T}} \\
& \tilde{M}_{d 1}:=\left[\bar{M}_{11}+\bar{M}_{12}, \ldots, \bar{M}_{q 1}+\bar{M}_{q 2}\right] ; \quad \Delta:=\left[\begin{array}{cc}
\mathcal{Y} & \mathcal{Y} \\
\mathcal{Y} & X
\end{array}\right] \\
& \tilde{M}_{d 2}:=\left[\bar{M}_{11}, \ldots, \bar{M}_{q 1}\right], \quad \tilde{B}_{d}:=\left[B_{1}, 0, B_{2}, 0, \ldots, B_{q}, 0\right] \\
& \hat{B}_{j}^{\mathrm{T}}:=\left[\begin{array}{cc}
B_{j}^{\mathrm{T}} \mathcal{Y} & B_{j}^{\mathrm{T}} X \\
0 & p_{j} D_{j}^{\mathrm{T}} \tilde{H}^{\mathrm{T}}
\end{array}\right] \\
& \Theta_{2}:=\operatorname{diag}\{\Delta, \Delta\} \\
& \tilde{D}_{d}:=\operatorname{diag}\left\{0, D_{1}^{\mathrm{T}} \tilde{H}^{\mathrm{T}}, 0, D_{2}^{\mathrm{T}} \tilde{H}^{\mathrm{T}}, \ldots, 0, D_{q}^{\mathrm{T}} \tilde{H}^{\mathrm{T}}\right\} \\
& \Theta_{1}:=\operatorname{diag}\left\{\Pi, \Delta, \operatorname{diag}_{q}\{\Delta\}, \Pi, \Delta\right\}
\end{aligned}
$$

then the system (7) is exponentially mean-square stable. The parameters of the filter $\left(\Sigma_{f}\right)$ are given as follows:

$$
G:=(\mathcal{Y}-X)^{-1} \tilde{G} \quad H:=(\mathcal{Y}-X)^{-1} \tilde{H} .
$$

\section{ILLUSTRATIVE EXAMPLE}

Consider a discrete-time NCS (1)-(3) with initial state $\left[\begin{array}{ll}0.01 & -0.3\end{array}\right]^{\mathrm{T}}$ and other system data given as follows:

$$
\begin{array}{rlrl}
q & =2 d_{1}=1 & d_{2}=3 & p_{0}=0.4 p_{1}=0.6 p_{2}=0.9 \\
A & =\left[\begin{array}{cc}
0.1 & 0 \\
-0.02 & 0.2
\end{array}\right] & B_{1}=\left[\begin{array}{cc}
0.12 & 0 \\
0 & 0.1
\end{array}\right] \\
B_{2} & =\left[\begin{array}{cc}
0.1 & 0.0512 \\
0 & 0.1
\end{array}\right] & C=\left[\begin{array}{cc}
0.02 & 0 \\
0 & 0.01
\end{array}\right] \\
D_{1} & =\operatorname{diag}\{-0.1,0.1\} & D_{2}=-0.1 I_{2} & \\
E & =\left[\begin{array}{ll}
0.08 & 0.07 \\
0.01 & 0.14
\end{array}\right] & F=\left[\begin{array}{cc}
0.13 & 0.09 \\
0.01 & 0.16
\end{array}\right] \\
K_{1} & =\operatorname{diag}\{0.35,0.35\} & K_{2}=\operatorname{diag}\{0.35,0.35\} \\
L_{1} & =\operatorname{diag}\{0.3,0.3\} & L_{2}=\operatorname{diag}\{0.3,0.3\} .
\end{array}
$$

Using MATLAB LMI-control Toolbox to solve (29) and (30), we can calculate the filter parameters as follows:

$$
G=\left[\begin{array}{cc}
0.1217 & 0.0009 \\
-0.0401 & 0.3059
\end{array}\right] \quad H=\left[\begin{array}{ll}
0.4559 & 0.2520 \\
0.2835 & 0.7126
\end{array}\right]
$$

\section{REFERENCES}

[1] Y. Cao, Z. Lin, and B. M. Chen, "An output feedback $H_{\infty}$ controller design for linear systems subject to sensor nonlinearities," IEEE Trans. Circuits Syst. I, Fundam. Theory Appl., vol. 50, no. 7, pp. 914-921, Jul. 2003.

[2] Q. L. Han, "Absolute stability of time-delay systems with sector-bounded nonlinearity," Automatica, vol. 41, no. 12, pp. 2171-2176, Dec. 2005

[3] X. He, Z. Wang, and D. Zhou, "Robust $H_{\infty}$ filtering for networked systems with multiple state delays," Int. J. Control, vol. 80, no. 8, pp. 1217-1232, Aug. 2007.

[4] H. Gao and C. Wang, "Robust $L_{2} / L_{\infty}$ filtering for uncertain systems with multiple time-varying state delays," IEEE Trans. Circuits Syst. I, Fundam. Theory Appl., vol. 50, no. 4, pp. 594-599, Apr. 2003.

[5] H. Gao, J. Lam, C. Wang, and S. Xu, "Control for stability and positivity: Equivalent conditions and computation," IEEE Trans. Circuits Syst. II, Exp. Briefs, vol. 52, no. 9, pp. 540-544, Sep. 2005.

[6] H. Gao and C. Wang, "A delay-dependent approach to robust $H_{\infty}$ filtering for uncertain discrete-time state-delayed systems," IEEE Trans. Signal Process., vol. 52, no. 6, pp. 1631-1640, Jun. 2004.

[7] E. Gershon, U. Shaked, and I. Yaesh, $H_{\infty}$ Control and Estimation of State-Multiplicative Linear Systems. Berlin, Germany: SpringerVerlag, 2005, Lecture Notes in Control and Information Sciences.

[8] G. P. Liu, Y. Xia, D. Rees, and W. S. Hu, "Design and stability criteria of networked predictive control systems with random network delay in the feedback channel," IEEE Trans. Syst., Man, Cybern. C, Appl. Rev., vol. 37, no. 2, pp. 173-184, Mar. 2007.

[9] Y. S. Moon, P. Park, W. H. Kwon, and Y. S. Lee, "Delay-dependent robust stabilization of uncertain state-delayed systems," Int. J. Control, vol. 74, no. 14, pp. 1447-1455, Sep. 2001.

[10] S. K. Nguang and W. Assawinchaichote, " $H_{\infty}$ filtering for fuzzy dynamical systems with D stability constraints," IEEE Trans. Circuits Syst. I, Fundam. Theory Appl., vol. 50, no. 11, pp. 1503-1508, Nov. 2003.

[11] A. V. Savkin and T. M. Cheng, "Detectability and output feedback stabilizability of nonlinear networked control systems," IEEE Trans. Autom. Control, vol. 52, no. 4, pp. 730-735, Apr. 2007.

[12] L. Schenato, B. Sinopoli, M. Franceschetti, K. Poolla, and S. S. Sastaty, "Foundations of control and estimation over lossy networks," Proc. IEEE, vol. 95, no. 1, pp. 163-187, Jan. 2007.

[13] X. M. Sun, J. Zhao, and D. J. Hill, "Stability and $L_{2}$-gain analysis for switched delay systems: A delay-dependent method," Automatica, vol. 42, no. 10, pp. 1769-1774, Oct. 2006.

[14] Z. Wang, D. W. C. Ho, and X. Liu, "Robust filtering under randomly varying sensor delay with variance constraints," IEEE Trans. Circuits Syst. II, Exp. Briefs, vol. 51, no. 6, pp. 320-326, Jun. 2004.

[15] Z. Wang, F. Yang, D. W. C. Ho, and X. Liu, "Robust $H_{\infty}$ filtering for stochastic time-delay systems with missing measurements," IEEE Trans. Signal Process., vol. 54, no. 7, pp. 2579-2587, Jul. 2006. 